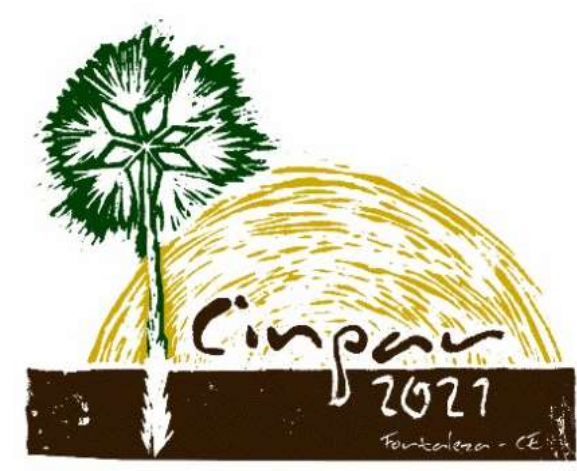

XVII Congresso Internacional sobre Patologia e

Reabilitação das Construções

XVII Congreso Internacional sobre Patología y Rehabilitación de las Construcciones

XVII International Conference on Pathology and Constructions Rehabilitation

FORTALEZA (Brasil), 3 a 5 de junho de 2021

https://doi.org/10.4322/CINPAR.2021.028

\title{
Análise dos efeitos da substituição do agregado miúdo por resíduo de vidro moído de tipo Long Neck marrom em blocos de concreto
}

\section{Analysis of the effects of replacing fine aggregate with brown long neck ground glass residue on concrete blocks}

\author{
Matheus Arthur Bazzan LOTTI' ${ }^{1}$, Marinês LANGOSKI² \\ ${ }^{1}$ Universidade Regional Integrada, URI, Erechim, RS, Brasil, matheus.lotti@hotmail.com \\ ${ }^{2}$ Universidade Regional Integrada, URI, Erechim, RS, Brasil, engenheiraml@hotmail.com
}

\begin{abstract}
Resumo: Atualmente existe um problema relacionado à reciclagem das embalagens de vidro das garrafas Long Neck, uma vez que estas não têm um destino adequado por não serem economicamente viáveis. Com isso, nesta pesquisa estudou-se a possibilidade da utilização do resíduo de vidro moído de Long Neck em substituição parcial ao agregado miúdo (areia), recurso natural utilizado na fabricação de blocos de concreto. Para isso realizou-se a caracterização dos materiais que foram utilizados para confeç̧ão dos blocos de referência (0\%), e definiram-se a granulometria do vidro moído que foi substituído, já em porcentagens definidas de $10 \%$ e $25 \%$. Para métodos comparativos trabalhou-se com uma empresa parceira com sede no município de Mariano Moro - RS, que oportunizou sua estrutura para a produção dos blocos de concreto. Portanto, foram realizados diferentes ensaios para avaliar a influência da substituição do agregado nas propriedades do bloco no estado endurecido (análise dimensional, índice de absorção da água, peso específico e resistência à compressão). Analisando os resultados, sobre análise dimensional, todos os blocos tiveram resultados satisfatórios, porém as dimensões de altura e largura ficaram um pouco acima do exigido por norma; pelo índice de absorção de água todas as composições atenderam a normativa, sendo o teor de substituição 10 \% com menor absorção. Para o peso específico o melhor resultado foi obtido para o bloco de teor $25 \%$, com menor valor de $24,52 \mathrm{kN} / \mathrm{m}^{3}$; em relação ao ensaio de resistência à compressão o único bloco que atingiu o determinado por pela NBR 6136 (2016) foi o de teor $25 \%$, com 3,15 MPa. Com isso, com os resultados obtidos nos ensaios, foi possível concluir a viabilidade da substituição no bloco de concreto com teor 25\%, pois atingiu todas as exigências da NBR 6136 (2016).
\end{abstract}

Palavras-chave: Garrafas Long Neck, areia, bloco de concreto.

Abstract: Currently, there is a problem related to the recycling of glass packaging for Long Neck bottles, since they do not have an adequate destination because they are not economically viable. Thus, in this research, the possibility of using the crushed Long Neck glass residue to partially replace the fine aggregate (sand), a natural resource used in the manufacture of concrete blocks, was studied. For this, the characterization of the materials that were used to make the reference blocks (0\%) was carried out, and the granulometry of the ground glass that was replaced was defined, already in defined percentages of $10 \%$ and $25 \%$. For comparative methods, we worked with a partner company based in the municipality of Mariano Moro - RS, which provided its structure for the production of concrete blocks. Therefore, different tests were carried out to evaluate the influence of aggregate substitution on the properties of the block in the hardened state (dimensional analysis, water absorption index, specific weight and resistance to compression). Analyzing the results, on dimensional analysis, all the blocks had satisfactory results, however the dimensions of height and width were slightly 
above that required by the norm; due to the water absorption index, all compositions complied with the regulations, the substitution content being $10 \%$ with less absorption. For the specific weight, the best result was obtained for the $25 \%$ content block, with a lower value of $24.52 \mathrm{kN} / \mathrm{m}^{3}$; in relation to the compressive strength test, the only block that reached the level determined by NBR 6136 (2016) was 25\%, with $3.15 \mathrm{MPa}$. Thus, with the results obtained in the tests, it was possible to conclude the feasibility of substitution in the concrete block with 25\% content, as it reached all the requirements of NBR 6136 (2016).

Keywords: Long Neck bottles, sand, concrete block.

\section{Introdução}

De acordo com o Conselho Internacional da Construção - $\mathrm{ClB}$, a indústria da construção é o setor de atividades que mais consome recursos naturais e utiliza energia de forma intensiva, gerando consideráveis impactos ambientais. Além dos impactos relacionados ao consumo de matéria e energia, há aqueles associados à geração de resíduos sólidos, líquidos e gasosos. Estima-se que mais de $50 \%$ dos resíduos sólidos gerados pelo conjunto das atividades humanas sejam provenientes da construção. Tais aspectos ambientais, somados à qualidade de vida que o ambiente construído proporciona, sintetizam as relações entre construção e meio ambiente (Ministério do Meio Ambiente - MMA, 2016).

Com isso, quando o assunto recursos naturais é discutido, várias questões são analisadas, seja pelo possível esgotamento no nosso meio ou pelas consequências causadas pela sua retirada do ambiente. A areia, quando extraída de encostas de rios gera problemas ambientais. Estudos realizados verificaram várias consequências devido a sua retirada, sendo que os impactos de maior significância foram incidência de processos erosivos, danos a fauna e a flora terrestres, depreciação da qualidade da água, alterações na geomorfologia fluvial e o aumento da oferta do produto (NOGUEIRA, 2016).

Segundo Andrade (2017), promover a inovação com o foco em sustentabilidade se torna mais importante a cada dia. As necessidades do meio ambiente devem ser levadas em consideração se pensarmos em novas formas de produção capazes de trazer benefícios tanto ao meio ambiente quanto às organizações. $\mathrm{Na}$ construção civil, vários estudos estão direcionados nessa área por ser um grande causador desses danos, como extração exagerada de recursos naturais ou a falta de destino adequado de materiais, os quais acabam causando prejuízos ao meio ambiente. Por esses motivos, o presente estudo propõe uma solução viável às ações citadas, substituindo o meio natural pelo material sem descarte.

Como contribuição à estudos da área, foi substituído o agregado miúdo por vidro moído em blocos de concreto, que tem as características do concreto convencional, além de ter uma aplicabilidade maior na construção civil, pois sua fabricação proporciona menos variabilidade de traços e ambientes de fabricação estabilizados, originando um controle mais acessível do vidro em comparação a produção de concreto.

\section{Referencial Teórico}

\subsection{Blocos de concreto}

Os blocos de concreto são industrializados e compostos basicamente por cimento Portland, agregados graúdos e miúdos, água e, ainda, adições ou aditivos que lhe proporcionam as características desejadas no bloco (RAMALHO; CORRÊA, 2003).

Os blocos de concreto amparados pela NBR 6136 (2016) possuem formato e dimensões padronizadas, que proporcionam um sistema construtivo limpo, prático, rápido, econômico e eficiente. Além disso, o material concreto possui um módulo de elasticidade similar ao da junta de argamassa, aproximando a resistência da alvenaria à do bloco (COMUNIDADE DA CONSTRUÇÃO, 2019). 
No Brasil, grande parte das fábricas não possuem um controle rigoroso sobre o processo produtivo que confira qualidade e segurança para as unidades produzidas. São poucas as fábricas que possuem o selo de qualidade da Associação Brasileira de Cimento Portland (ABCP).

\subsection{Vidros}

O vidro é um material inorgânico, cujo elemento básico é a sílica encontrada em abundância na areia, que se une a óxidos fundentes, estabilizantes e substâncias corantes para formar o silicato (DA ROSA et al., 2007).

As embalagens de cerveja tipo Long Neck ou one way são consideradas hoje um dos mais problemáticos resíduos gerados no mundo, pois após o consumo da bebida, são simplesmente descartadas, ocupando espaço do destino final.

Essas embalagens são fabricadas para atender aos interesses das indústrias vidreiras e as indústrias envasadoras de bebidas, visando a competitividade entre as embalagens, especificamente entre o alumínio e o vidro sem pensar nas consequências da poluição causada ao meio ambiente, comprometendo a qualidade de vida e a segurança de todos.

Para deixar este tipo de embalagem competitiva junto ao alumínio, a indústria vidreira retirou alguns componentes químicos que davam certo peso à embalagem, ficando comprometida a sua resistência, não permitindo o retorno para um segundo envase, ou seja, ela só vai ao consumidor não retornando para ser reutilizada, passando a ser um problema ambiental (FUNVERDE, 2009).

\subsubsection{Impacto ambiental e reciclagem}

O descarte inadequado do material pela população ocasiona o acúmulo em vias públicas, ocasionando problemas em bueiros, bocas de lobos, riachos ou em aterros sanitários que acabam ocupando espaço nesses depósitos que poderiam ser utilizados por materiais orgânicos de rápida decomposição. Em alguns casos o mal armazenamento ocasiona focos de doenças como a dengue, o zika vírus e o chikungunya, agravando o problema ambiental.

O vidro, entre os materiais de uso mais frequente em embalagens, é provavelmente o que apresenta maior facilidade para a reciclagem. Com efeito, o vidro, em termos teóricos, é $100 \%$ reciclável, e a mesma "unidade" de vidro pode ser aproveitada inúmeras vezes (DA ROSA et al., 2007).

As embalagens de vidro destinadas a alguns segmentos, como o de bebidas, podem ser não apenas recicláveis, mas também retornáveis, o que significa que a mesma embalagem passa por vários ciclos de utilização. Tais embalagens são normalmente garrafas de certo peso, projetadas para ter resistência mecânica adequada ao longo de sua vida útil (DA ROSA et al., 2007).

Por sua vez, as garrafas Long Neck que não são consideradas para reutilização e nem reciclagem por não serem economicamente viáveis, sendo mais oportuno para as fábricas retirar novamente as matérias-primas da natureza.

\subsubsection{Utilização do vidro em blocos de concreto}

Com o promissor futuro baseado na importância do uso da sustentabilidade, pesquisadores tem buscado aprofundamento de estudos em materiais ambientalmente corretos ou com diminuição no impacto na sua fabricação e utilização, mas que não prejudiquem as características do mesmo.

Os autores Serpa et al., (2012), analisaram em sua pesquisa o comportamento do vidro reciclado em materiais cimentícios e os resultados obtidos nesta campanha experimental mostram que o vidro reciclado apresenta potencial como agregado ou como adição mineral, embora a sua influência na RAA (reação álcaliagregado) dependa do teor de substituição e da granulometria em que é empregue. 
A autora Bottega (2017), substituindo o agregado concluiu com a análise de todos os resultados obtidos nos ensaios, que o melhor teor de substituição a ser utilizado é o teor de $50 \%$, pois incorpora mais resíduo de vidro na composição do concreto, melhorando as suas propriedades mecânicas, sendo também ambientalmente recomendável.

A carência de estudos que tratam da substituição de vidro moído por areia em bloco de concreto, aumenta a importância e expectativa nos resultados desse estudo.

\section{Metodologia de pesquisa}

A metodologia desta pesquisa procura demonstrar as propriedades dos materiais através de resultados obtidos na caracterização física, química e mecânica e procedimentos utilizados para a execução dos ensaios do programa experimental.

A caracterização dos materiais e os ensaios foram executados no Laboratório de Preparo de Amostras e Técnicas Construtivas (LAPATEC), do curso de Engenharia Civil, da URI - Campus de Erechim e no Laboratório de Precisão Analítica, da URI - Campus de Erechim. A Figura 1 demonstra o Programa Experimental da pesquisa.

Figura 1 - Programa experimental.

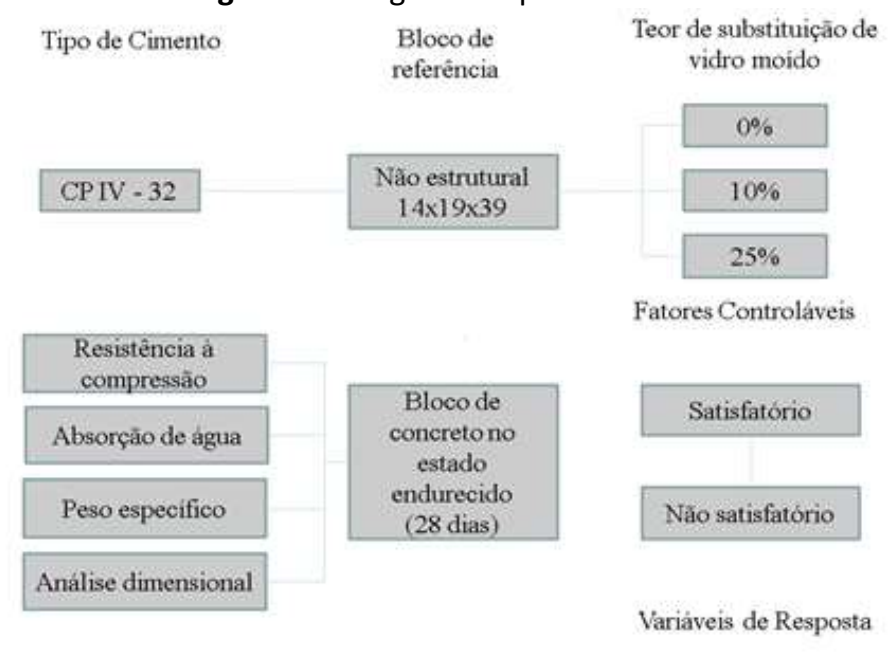

Fonte: Autores (2021).

Para execução dos ensaios os procedimentos e a quantidade de corpos-de-prova são determinados por norma, e estão demonstrados no Quadro 1.

Quadro 1 - Propriedades avaliadas, normativas e número de corpos-de-prova (cps)

\begin{tabular}{|c|c|c|}
\hline Propriedades avaliadas & Normas (NBR) & Número de cps por percentual de substituição \\
\hline $\begin{array}{c}\text { Resistência à } \\
\text { compressão }\end{array}$ & $12118(2013)$ & 12 \\
\hline Absorção de água & $12118(2013)$ & 3 \\
\hline Peso específico & $15961-1(2011)$ & 3 \\
\hline Análise dimensional & $6136(2016)$ & 6 \\
\hline
\end{tabular}

Fonte: Autores (2021). 


\section{Resultados}

\subsection{Análise dimensional}

Realizados os procedimentos para determinação das medidas dos blocos, aplicou-se a média aritmética para obtenção dos resultados e comparação com as exigências na NBR 6136 (2016), como demonstrado no Quadro 2.

\begin{tabular}{|c|c|c|c|c|c|c|c|}
\hline \multicolumn{8}{|c|}{ Quadro 2 - Analıse dımensıonal dos blocos } \\
\hline $\begin{array}{l}\text { Percentual de } \\
\text { substituição }\end{array}$ & $\begin{array}{l}\text { Largura } \\
\text { (mm) }\end{array}$ & $\begin{array}{l}\text { Altura } \\
(\mathrm{mm})\end{array}$ & $\begin{array}{c}\text { Comprimento } \\
(\mathrm{mm})\end{array}$ & $\begin{array}{c}\text { Parede } \\
\text { longit. (mm) }\end{array}$ & $\begin{array}{c}\text { Parede } \\
\text { transv. }(\mathrm{mm})\end{array}$ & $\begin{array}{l}\text { Furo longit } \\
\qquad(\mathrm{mm})\end{array}$ & $\begin{array}{l}\text { Furo transv. } \\
\text { (mm) }\end{array}$ \\
\hline $0 \%$ & 142,48 & 193,17 & 390,11 & 28,79 & 26,02 & 161,23 & 86,45 \\
\hline $10 \%$ & 142,47 & 193,50 & 390,06 & 29,06 & 26,08 & 160,52 & 86,27 \\
\hline $25 \%$ & 142,45 & 193,44 & 390,22 & 29,03 & 26,13 & 160,42 & 86,26 \\
\hline
\end{tabular}

Fonte: Autores (2021).

Apesar das medidas estatísticas enquadrarem todas as amostras como homogêneas, a média das dimensões da largura e altura, em todos os traços ensaiados, ultrapassou os limites estabelecidos em norma, uma vez que a mesma estabelece uma tolerância na faixa de largura que varia entre $138 \mathrm{~mm}$ e $142 \mathrm{~mm}$, e para altura de $187 \mathrm{~mm}$ e $193 \mathrm{~mm}$. Os limites ultrapassados podem ser originados pelo processo de fabricação, como a má higienização da prensa, provocando assim uma prensagem maior do que o esperado devido ao acúmulo de concreto na face de prensagem, ou pelo equipamento estar no momento de ter manutenção, podendo assim ter falta de lubrificação na face de prensagem, provocando uma possível perda de calibração na vibroprensa e um possível desgaste sofrido pela forma da vibro-prensa.

No que se refere aos valores de comprimento nominal do bloco, verificou-se, que todos blocos atenderam ao comprimento estabelecido por norma, pois o recomendado varia entre 387,00mm e 393,00mm.

As espessuras das paredes, tanto longitudinais como transversais superaram as exigências mínimas de 18 $\mathrm{mm}$; as dimensões dos furos também atenderam as especificações.

\subsection{Absorção de água}

Os valores obtidos para o ensaio de absorção de água, por traço de substituição estão demonstrados no Quadro 3.

Quadro 3-Resultados de absorção dos blocos

\begin{tabular}{|c|c|c|c|}
\hline $\begin{array}{c}\text { Percentual de } \\
\text { substituição }\end{array}$ & Absorção (\%) & $\begin{array}{c}\text { Desvio padrão } \\
\text { (\%) }\end{array}$ & $\begin{array}{c}\text { Coeficiente de } \\
\text { variação (\%) }\end{array}$ \\
\hline $0 \%$ & 7,10 & 0,12 & 1,74 \\
\hline $10 \%$ & 6,96 & 0,21 & 3,01 \\
\hline $25 \%$ & 7,23 & 0,19 & 2,62 \\
\hline
\end{tabular}

Em análise dos resultados demonstrados no Quadro 3 foi possível inferir que os valores médios obtidos atenderam o proposto pela NBR 6136 (2016), a qual estabelece um limite de 10\% para o percentual de absorção. 
Em análise estatística no programa ANOVA, verificou-se que os percentuais de substiuição adotados $(0,10$ e 25\%) não apresentaram resultados significativos na propriedade avaliada absorção de água.

\subsection{Peso específico}

Os resultados obtidos para o peso específico para cada percentual de substituição estão demonstrados no Quadro 4.

Quadro 4 - Peso específico dos blocos

\begin{tabular}{|c|c|c|c|c|c|}
\hline $\begin{array}{l}\text { Percentual de } \\
\text { substituição }\end{array}$ & $\begin{array}{l}\text { Massa média dos } \\
\text { blocos secos (g) }\end{array}$ & $\begin{array}{l}\text { Volume médio } \\
\qquad\left(\mathrm{cm}^{3}\right)\end{array}$ & $\begin{array}{c}\text { Peso específico } \\
\left(\mathrm{g} / \mathrm{cm}^{3}\right)\end{array}$ & $\begin{array}{l}\text { Desvio padrão } \\
\qquad\left(\mathrm{g} / \mathrm{cm}^{3}\right)\end{array}$ & $\begin{array}{c}\text { Coeficiente de } \\
\text { variação (\%) }\end{array}$ \\
\hline $0 \%$ & 14566,67 & 5715,2 & 2,55 & 0,10 & 3,98 \\
\hline $10 \%$ & 14366,67 & 5715,2 & 2,51 & 0,08 & 3,04 \\
\hline $25 \%$ & 14300,00 & 5715,2 & 2,50 & 0,04 & 1,52 \\
\hline
\end{tabular}

Fonte: Autores (2021).

De acordo com a NBR 15961-1 (2011), o peso específico médio dos blocos de concreto sem uma avaliação precisa é de $14 \mathrm{KN} / \mathrm{m}^{3}$. Tendo em vista o proposto, os traços dos blocos de concreto produzidos estão acima do recomendado, pois atingiram um peso específico médio de $25,01 \mathrm{kN} / \mathrm{m}^{3}, 24,61 \mathrm{kN} / \mathrm{m}^{3}$ e $24,52 \mathrm{kN} / \mathrm{m}^{3}$, respectivamente aos traços com $0 \%, 10 \%$ e $25 \%$ de substituição de vidro moído.

\subsection{Resistência à compressão}

Os resultados obtidos para a resistência à compressão, para cada percentual de substituição estão demonstrados no Quadro 5.

Quadro 5 - Resultados de resistência à compressão

\begin{tabular}{|c|c|c|c|}
\hline $\begin{array}{c}\text { Percentual de } \\
\text { substituição }\end{array}$ & $\begin{array}{c}\text { Resistência } \\
\text { média (MPa) }\end{array}$ & $\begin{array}{c}\text { Desvio padrão } \\
\mathbf{( \% )}\end{array}$ & $\begin{array}{c}\text { Coeficiente de } \\
\text { variação (\%) }\end{array}$ \\
\hline $0 \%$ & 2,36 & 0,44 & 18,71 \\
\hline $10 \%$ & 2,41 & 0,21 & 8,69 \\
\hline $25 \%$ & 3,15 & 0,33 & 10,40 \\
\hline
\end{tabular}

Fonte: Autores (2021).

A Figura 2 mostra a influência do teor de substituição de resíduo de vidro moído sobre a resistência à compressão dos blocos de concreto, a partir da análise estatística (ANOVA). 
Figura 2 - Resultados de resistência à compressão

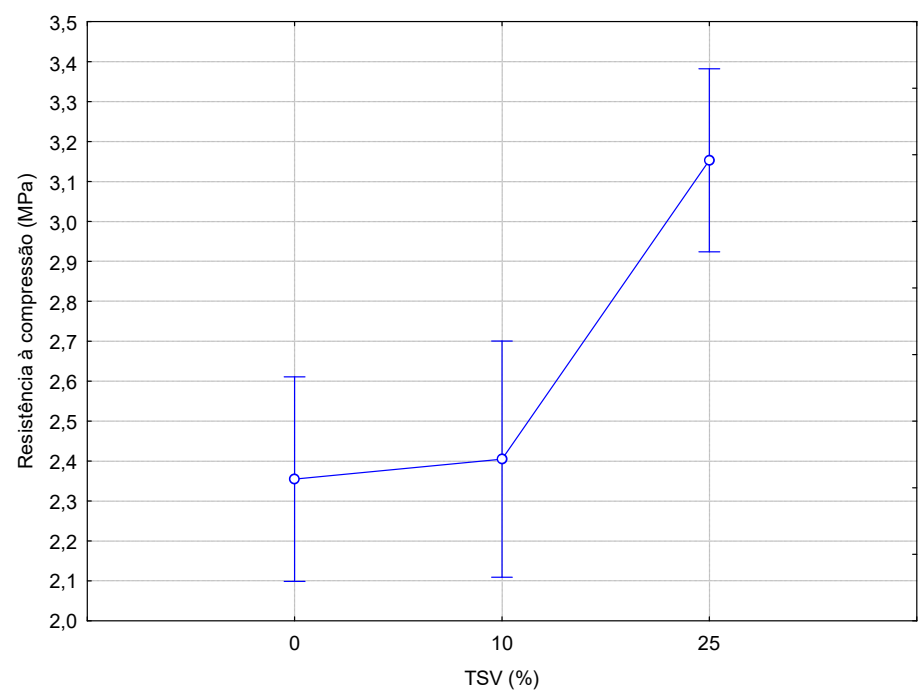

TSV= Teor de substituição de vidro moído Fonte: Autores (2021).

Analisando a Figura 2 é possível inferir que o aumento significativo de resistência foi obtido para o traço com $25 \%$ de substituição, em relação ao traço referência. Não foi observada diferença significativa para o traço com $10 \%$ de substituição, em relação ao traço sem substituição.

Para Righi et al., (2011), tal comportamento pode ser justificado pelo resultado do aumento no teor de finos da mistura quando comparado com a curva granulométrica da areia utilizada. Outra justificativa para o aumento com a substituição pode ser a composição do vidro, ao passo que, o material é essencialmente composto por sílica amorfa e outros componentes que podem vir a melhorar a durabilidade da matriz como adição mineral pelo seu potencial pozolânico (PAIVA, 2009).

\section{Conclusões}

Em análise de todos os resultados obtidos nos ensaios, o melhor teor de substituição a ser utilizado é o teor de $25 \%$, pois incorpora mais resíduo de vidro na composição do bloco de concreto, somado aos maiores valores de resistência à compressão, sendo também ambientalmente recomendável.

Estudando os requisitos da norma pode-se inferir que somente o bloco com $25 \%$ de substituição pode ser usado na construção civil, pois foi o único cuja resistência à compressão ultrapassou o valor mínimo de 3 MPa recomendados por norma. Sua utilização pode ser em muros, alvenarias sem função estrutural, todas demandas de projetos que solicitam blocos de concreto classificados pela classe C da NBR 6136 (2016), dando relevância que somente com a substituição do vidro pela areia, sem alterar traço e um evidente aumento de custos na produção, atingiu o exigido para utilização.

Nesta pesquisa o reaproveitamento do resíduo de vidro, proveniente da moagem de garrafas long neck, na indústria da construção civil se mostrou viável, considerando que quando foi utilizado na produção de blocos de concreto demonstrou resultados satisfatórios, ao serem comparados com os corpos de prova de referência. A utilização desse resíduo evita o descarte incorreto, tornando os blocos de concreto produzidos com a substituição parcial do agregado miúdo um material mais resistente e de construção sustentável. 


\section{Referências Bibliográficas}

Andrade, V. Blog Way Carbon. Net, 2017. Disponível em http://blog.waycarbon.com. Acesso em 25 de abril de 2019.

ASSOCIAÇÃO BRASILEIRA DE NORMAS TÉCNICAS. NBR 6136: Blocos vazados de concreto simples para alvenaria - requisitos. Rio de Janeiro, 2016.

ASSOCIAÇÃO BRASILEIRA DE NORMAS TÉCNICAS. NBR 12118: Blocos vazados de concreto simples para alvenaria - métodos de ensaio. Rio de Janeiro, 2013.

ASSOCIAÇÃO BRASILEIRA DE NORMAS TÉCNICAS. NBR 15961-1: Alvenaria Estrutural - Blocos de concreto Parte 1: Projeto. Rio de Janeiro, 2011.

Bottega, S. P. B. Análise dos efeitos da substituição parcial do agregado miúdo por resíduo de vidro moído no concreto convencional. 2017. 64 f. Projeto final de curso de bacharel em Engenharia Civil. Universidade Regional Integrada do Alto Uruguai e das Missões. Erechim, RS.

Comunidade da Construção. Net, 2019. Disponível em http://www.comunidadedaconstrucao.com.br. Acesso em 10 de maio de 2019.

Da Rosa, S. E. S.; Cosenza, J. P.; Barroso, D. V. Considerações sobre a indústria e o vidro no Brasil. Net, 2007. Disponível em http://web.bndes.gov.br. Acesso em 05 de junho de 2019.

Funverde. A poluição causada pelas embalagens long neck e a lei 333/09. Net, 2009. Disponível em http://www.funverde.org.br. Acesso em 16 de abril de 2019.

Ministério do Meio Ambiente (MMA). Disponível em http://www.mma.gov.br. Acesso em 15 de abril de 2019.

Nogueira, G. R. F. A extração de areia em cursos d'água e seus impactos: proposição de uma matriz de interação. 2016. 74 f. Trabalho de conclusão de curso (Graduação em Engenharia Ambiental e Sanitária) - Universidade Federal de Juiz de Fora. UFJF, 2016.

Paiva, O. A. Resíduo industrial de vidro moído em argamassa de cimento Portland. 2009. Dissertação de Mestrado. Universidade Federal do Amazonas. Manaus, 2009.

Ramalho, M. A.; Corrêa, M. R. S. Projeto de edifícios de alvenaria estrutural. 1 ed. São Paulo: Editora Pini, 174 p, 2003.

Righi, D.; Kohler, L.; Kirchhof, L.; Lima, R. Efeitos da substituição de areia por vidro moído no comportamento de concretos em elevadas temperaturas. 2011. Revista Engenharia Estudo e Pesquisa. V. 11, n. 2, p. 2835. Rio de Janeiro, 2011.

Serpa, D.; Silva, S. A.; De Brito, J.; Pontes, J.; Soares, D. 2012. Trabalho de Conclusão de Curso. Comportamento do vidro reciclado em materiais cimentícios. Universidade técnica de Lisboa. Lisboa. 